\title{
Hydrogen Adsorption in Carbon Nanostructures: Comparison of Nanotubes, Fibers, and Coals
}

\author{
H. Gijs Schimmel, ${ }^{[a]}$ Gordon J. Kearley, ${ }^{[a]}$ Marije G. Nijkamp, ${ }^{[b]}$ Cornelis T. Visser, ${ }^{[b]}$ \\ Krijn P. de Jong, ${ }^{[b]}$ and Fokko M. Mulder*[a]
}

\begin{abstract}
Single-walled carbon nanotubes (SWNT) were reported to have record high hydrogen storage capacities at room temperature, indicating an interaction between hydrogen and carbon matrix that is stronger than known before. Here we present a study of the interaction of hydrogen with activated charcoal, carbon nanofibers, and SWNT that disproves these earlier reports. The hydrogen storage capacity of these materials correlates with the surface area of the material, the activated charcoal having the largest. The SWNT appear
\end{abstract}

to have a relatively low accessible surface area due to bundling of the tubes; the hydrogen does not enter the voids between the tubes in the bundles. Pressure-temperature curves were used to estimate the interaction potential, which was found to be $580 \pm 60 \mathrm{~K}$. Hydrogen gas was adsorbed in amounts up to

Keywords: carbon - density functional calculations • hydrogen adsorption • physisorption • single walled nanotubes
$2 \mathrm{wt} \%$ only at low temperatures. Molecular rotations observed with neutron scattering indicate that molecular hydrogen is present, and no significant difference was found between the hydrogen molecules adsorbed in the different investigated materials. Results from density functional calculations show molecular hydrogen bonding to an aromatic $\mathrm{C}-\mathrm{C}$ bond that is present in the materials investigated. The claims of high storage capacities of SWNT related to their characteristic morphology are unjustified.

\section{Introduction}

The importance of hydrogen as a future energy carrier is generally acknowledged. The advantages of a hydrogen-based economy would be its sustainable and environmentally friendly character. Apart from factors concerning the energy-efficient production of hydrogen as well as cost-effective use of hydrogen in fuel cells, the application of hydrogen as an energy carrier is limited by hydrogen storage problems. Storage of hydrogen is either too expensive, too heavy, takes too much volume and/or is unsafe. Therefore hydrogen storage in new materials and devices is an active field for research worldwide. Storage of hydrogen in new forms of carbon materials attracted worldwide interest when Dillon et al. published 5-10 wt \% hydrogen storage in single-walled

[a] Dr. F. M. Mulder, H. G. Schimmel, Prof. G. J. Kearley Interfaculty Reactor Institute, Delft University of Technology Mekelweg 15, 2629, JB Delft (The Netherlands) Fax: (+31)15-2788303

E-mail: f.m.mulder@iri.tudelft.nl

[b] Dr. M. G. Nijkamp, C. T. Visser, Prof. K. P. de Jong Inorganic Chemistry and Catalysis, Debye Institute Utrecht University P.O. Box 80083, 3508 TB Utrecht (The Netherlands) carbon nanotubes (SWNT). ${ }^{[1]}$ Subsequently claims of high hydrogen storage capacities at room temperature and pressures higher than 100 bar in graphitic nanofibers (GNF) and lithium- and potassium-doped multiwalled carbon nanotubes were made. ${ }^{[2-4]}$ However, more recently several reports that falsify these claims have appeared..$^{[-8]}$ Despite considerable effort, computations do not support those high storage capacities on the basis of physisorption processes. ${ }^{[9-14]}$

Recently we reported adsorption capacities in the range $0-2.5 \mathrm{wt} \%$ at low temperatures and ambient pressure in various nanostructured carbon samples. ${ }^{[15]}$ The process responsible for storage under the low-temperature ambientpressure conditions is clearly physisorption. ${ }^{[16,17]}$ An important yet unanswered question is how the strength of the interaction resulting in physisorption varies between carbonbased materials with different topologies. Herein, we report on a study of the interactions between hydrogen and different carbon materials ranging from activated charcoal to single walled carbon nanotubes. In our measurements the rotation of a hydrogen molecule is used as a sensitive probe for the presence of the molecule and for interactions between surface and adsorbed hydrogen molecules at the microscopic/molecular level. These results are compared to macroscopic adsorption measurements as well as first-principles calculations. 


\section{Experimental Section and Principles}

Materials: Four different materials have been investigated in this study: Two activated carbons: AC Norit 990293 (NAC) and AC Norit GSX (GSX), a 'fishbone' carbon nano-fiber sample (GNF), and a sample consisting of single-walled carbon nanotubes (SWNT). Activated carbons are highly micro- and mesoporous carbon materials. Steam-activated carbons have been prepared from raw materials (e.g. peat, lignite, coal) and carbonized and reacted with steam at $1000^{\circ} \mathrm{C}$. In this way some of the carbon atoms are removed by gasification, which yields a very porous structure.

Graphitic nanofibers were grown by using a fixed-bed reactor. Nickel particles on a silica support (Aerosil 200) dissociate methane, forming hydrogen and carbon atoms that dissolve in the nickel particle. These carbon atoms diffuse through the nickel catalyst particle to form a carbon nanofiber. The silica support was removed in a refluxed boiling $\mathrm{KOH}$ solution. In the same manner a treatment with $\mathrm{HNO}_{3}$ was used to remove the Ni particles. ${ }^{[18]}$ The nanofibers used in this investigation had a fishbone arrangement of the graphite planes with respect to the fiber axis. The diameter of these fibers ranges between 20 and $30 \mathrm{~nm}$, with an average of $25 \mathrm{~nm}$. We loaded these fishbone fibers with $0.2 \mathrm{wt} \% \mathrm{Pd}$ nanoparticles using ion-exchange from a $\mathrm{Pd}\left(\mathrm{NH}_{3}\right)_{4} \mathrm{Cl}_{2}$ solution. ${ }^{[19]}$ This was done to open up the possibility to have atomic $\mathrm{H}$ in the sample, that may possibly even intercalate in between the graphite layers as has been speculated by others. ${ }^{[2-4]}$

The single-walled carbon nanotubes were obtained commercially (from Carbon Nanotechnologies Incorporated, Houston, USA). The nanotubes were produced by the HiPCo process in which $\mathrm{Fe}(\mathrm{CO})_{5}$ decomposes in a $\mathrm{CO}$ atmosphere. ${ }^{[20]}$ Our sample contains $17.5 \mathrm{wt} \%$, or less than 5 at \%, of leftover iron catalyst particles. The sample was characterized by X-ray diffraction, which showed no sharp peaks, indicating that no crystalline form of graphite or iron was present. Mössbauer spectroscopy revealed that the leftover catalyst particles are almost exclusively present in the form of $\mathrm{Fe}_{3} \mathrm{C}$. Transmission electron microscopy was used to investigate the diameter of the nanotubes and the iron carbide particles. The carbon nanotubes are packed with a smallest repetition distance of about $1.25 \mathrm{~nm}$, indicating that the size of the tubes is around $1.25 \mathrm{~nm}$, whereas the iron carbide particles are larger, with diameters up to $4 \mathrm{~nm}$. The surface area of the relatively low number of massive iron carbide particles is much smaller than that of the nanotubes, and any adsorption of $\mathrm{H}_{2}$ on these particles can therefore not be observed

Adsorption measurements: Samples were loaded in aluminum containers and mounted in a closed cycle refrigerator. The samples were evacuated to below $1 \mathrm{mPa}$ while heated to $323 \mathrm{~K}$ for at least $12 \mathrm{~h}$. Adsorption isotherms were recorded by opening a valve to a known volume of hydrogen gas with a known pressure, waiting for equilibrium and measuring the pressure, and repeating this procedure up to 1 bar. Temperature-pressure curves were measured inside the same system.

Inelastic neutron scattering: The samples under investigation were loaded in aluminum sample chambers, mounted into a cryogenic system and evacuated to below $1 \mathrm{mPa}$ while heated to $323 \mathrm{~K}$ for at least $12 \mathrm{~h}$. The sample thickness was such that up to $10 \%$ of the neutrons was scattered by the nuclei of the sample when hydrogen was loaded. The samples were cooled to $3.6 \mathrm{~K}$ and a background measurement was performed on the evacuated sample. Then the sample was heated to $77 \mathrm{~K}$ and hydrogen was loaded up to a pressure of $1 \mathrm{bar}$. This temperature and pressure were chosen because these are the reference conditions in our previous work. When insufficient hydrogen had been adsorbed for a good neutron spectrum, filling was continued at 1 bar at a temperature that was lowered by a few degrees (this enhances the adsorption capacity considerably). In

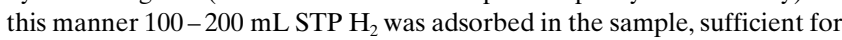
recording an accurate neutron spectrum in a reasonable time. The hydrogen loaded sample was then cooled down to $3.6 \mathrm{~K}$ and a spectrum was recorded. Note that we can exclude the possibility of having measured on solid hydrogen because the hydrogen adsorbs already completely at $50 \mathrm{~K}$ (i.e. the hydrogen vapor pressure is always much lower than the equilibrium vapor pressure for liquid or solid hydrogen).

The measurements were performed on the time-of-flight spectrometer RKS at the $2 \mathrm{MW}$ nuclear reactor in Delft (The Netherlands). This spectrometer uses two choppers and a rotating pyrolitic graphite mono- chromator to produce a pulsed, mono-energetic beam. The incident energy of the neutron beam was selected to be $157.3 \mathrm{~cm}^{-1}$. The scattered neutrons are detected by an array of detectors giving a momentum transfer range of $0.2-3.5 \AA^{-1}$ and their arrival time is recorded. The time-of-flight of the neutrons were transformed into energies and the structure factor $S(Q, \omega)$ was obtained by multiplying with $k_{i} / k_{f}$ and by normalizing on the incident beam monitor. To improve statistics the spectra were summed over the momentum transfer $Q$ so that the average value of the spectra is $1.6 \AA$. Scattering of the sample without hydrogen was subtracted from the spectrum of the hydrogen-loaded sample. In order not to extract too much of the 'empty sample spectrum', corrections were made for the small screening of the scattering of the sample holder and carbon sample by the hydrogen in the loaded sample. In this way only the scattering of hydrogen is visible in the corrected spectra. All experiments were performed at a temperature of $3.6 \mathrm{~K}$. These low temperatures are needed because at higher temperatures the thermal energy of the hydrogen molecules appears to enable them to move along the surface, giving unwanted broadening of the spectra.

To be able to observe a possible decrease in elastic scattering with time, each spectrum was divided into 5-10 sub-runs. Such decrease in elastic scattering could result from the slow conversion of ortho- to para-hydrogen because ortho-hydrogen scatters approximately 40 times more than parahydrogen (see below for explanation).

Theoretical calculations: To understand the experimental results we performed density functional theory calculations using $\mathrm{Dmol}^{3}$. We used the numerical basis set DND (double numerical with d functions) and the Perdew Wang local correlation density functional.

\section{The hydrogen molecule as a probe}

Due to its diatomic nature, hydrogen can rotate around two axes. The possible energies $E_{J}$ of the rotation of the hydrogen molecule are quantized and are labelled by the rotational quantum number $J$. For a hydrogen molecule that is free to rotate (this is in good approximation the case in solid hydrogen ${ }^{[21]}$ ) the energies of the rotational levels are given by $E_{J}=$ $J(J+1) B$. The rotational constant $B$, can be evaluated with the formula (1), in which $\hbar$ is Planck's constant divided by $2 \pi, m_{p}$ is the mass of the protons, and $R$ is the distance between the protons. ${ }^{[21]}$

$B=\frac{2 \hbar^{2}}{m_{p} R^{2}}$

From the experimental value of $B, 59.3 \mathrm{~cm}^{-1}(7.35 \mathrm{meV}),{ }^{[21]}$ we can evaluate the difference between the two lowest rotational states $(J=0$ and $J=1)$ : $119 \mathrm{~cm}^{-1}$ (or $\left.\approx 170 \mathrm{~K}\right)$. It is important to note here that the rotational transition only occurs for molecular hydrogen, that is atomic hydrogen bonded to a carbon plane does not show this transition.

Because the two protons in a $\mathrm{H}_{2}$ molecule are fermions, their overall wavefunction should be antisymmetric under exchange of the two nuclei. Rotational states with even values of $J$, have a symmetric spatial eigenfunction and the spin eigenfunction needs to be antisymmetric to achieve an overall antisymmetric wavefunction. This antisymmetric spin eigenfunction is a singlet spin state with nuclear spin 0 . Odd values of $J$ have an antisymmetric spatial eigenfunction, and conversely a symmetric spin part of the wavefunction (a triplet state with nuclear spin 1). Hydrogen in rotational states with odd values of $J$ is called ortho-hydrogen, whereas hydrogen in rotational states with even values of $J$ is denoted parahydrogen. At high temperatures (e.g. room temperature) the ratio of orthoto para-hydrogen molecules is 3 to 1 . At temperatures much lower than $170 \mathrm{~K}$ the lowest rotational state $J=0$ is energetically favorable. However the conversion from ortho- to para-hydrogen can be extremely slow because it involves a change of the total nuclear spin from 1 to 0 . The presence of paramagnetic catalysts or impurities as in our samples can speed up the conversion from days to less than hours. The neutron crosssection of ortho- and para-hydrogen are rather different (ortho: 80 barn molecule $^{-1}$, para: 1.8 barn molecule $^{-1}$ ). Care should be taken in the experiment to observe if the ortho-para conversion is complete or not. It may also be noted that atomic hydrogen has a large cross section $\left(80\right.$ barn atom $\left.^{-1}\right)$.

When a hydrogen molecule is adsorbed on a surface or in a cavity, the potential it feels may not be isotropic because of the atomic nature of any surface, and this may hinder the rotation of the adsorbed hydrogen molecule. Then spherical symmetry is broken, causing the triplet $J=1$ level to split. Such splitting contains information on the symmetry of the site at 
which hydrogen is adsorbed. An example was observed by e.g. Fitzgerald et al. for hydrogen molecules in interstitial sites in solid $\mathrm{C}_{60}{ }^{\left[{ }^{[2]}\right]}$

If the molecular hydrogen electron cloud is severely altered because it takes part in a bond with the surface, the distance between the protons increases due to reduced electronic screening. (This $\mathrm{H}-\mathrm{H}$ bond lengthening effect is also known as bond activation. ${ }^{[23]}$ ) This leads to a smaller value of the rotational constant $B$ (see [Eq. (1)]). Such effects of bond elongation were illustrated by Eckert and co-workers with experiments on different metal complexes having a dihydrogen ligand (the so-called Kubas compounds). They found that the separation of the lowest two rotational levels of the dihydrogen ligand is smaller in complexes where the dihydrogen ligand molecule was more tightly bound, and the larger proton - proton bond lengths found in this way were confirmed by neutron diffraction. ${ }^{[23]}$

For weakly bonded molecules, as is the case in physisorption by van der Waals interaction, no significant electron density is transferred to the adsorbent or is used to form the bond. Thus it is not expected that large shifts of the rotational constant $B$ will occur. The hydrogen molecules can then be described as (almost) free quantum-mechanical rotors. In contrast, for more strongly bound chemisorbed molecular hydrogen in for example zeolites a strong shift of the $J=1$ level can be observed. ${ }^{[24]}$

With the neutron experiments presented here we are able to discriminate between molecular and atomic hydrogen on the basis of the presence of the rotational transition and the strongly different neutron scattering cross sections. Furthermore under the assumption that the rotational level may be stronger split for stronger bound hydrogen, the value of the splitting gives an indication of the strength of the bond experienced by the hydrogen molecule. This should then also be in agreement with the adsorption energy found from the adsorption measurements. These methods will provide a means to investigate if the high storage capacity claims for carbon nanofibers and carbon nanotubes can be supported or not by a hydrogenmatrix interaction that is much stronger or of a different nature than found in other carbons.

\section{Results}

Pressure- and temperature-dependent hydrogen adsorption: All of these samples were tested for hydrogen storage capacity at $77 \mathrm{~K}$ and 1 bar. For the SWNT this was the first time that this was determined, while for the other compounds the adsorbed amounts (obtained volumetrically) under these conditions were the same as we found previously. ${ }^{[15]}$ Figure 1

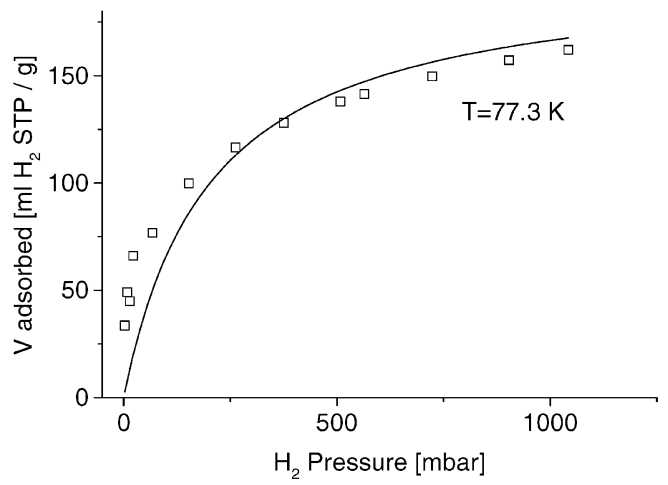

Figure 1. Hydrogen adsorption capacity for activated charcoal (Norit GSX) as a function of hydrogen pressure. The line is a fit with a Langmuir adsorption isotherm. The hydrogen surface coverage $\approx 0.8$ at $P=1$ bar.

shows the amount of adsorbed hydrogen as a function of hydrogen pressure for Norit GSX. The experimental curve follows the Langmuir isotherm (solid line in Figure 1) given by Equation (2), in which $\theta$ is the coverage of the adsorbent surface, $K$ is the equilibrium constant, and $P$ is the pressure. From the Langmuir isotherm a coverage of approximately 0.8 was derived.

$\theta=\frac{K P}{1+K P}$

We modified this model to get the temperature dependence of the pressure of the gas above a surface. Introducing the rate of adsorption, $\Phi_{a}$, which is proportional to the number of molecules hitting a vacant adsorption site we get Equation (3), where $T$ is the temperature of the system and $(1-\theta)$ is the fraction of unoccupied adsorption sites.

$\Phi_{a} \propto \frac{P}{\sqrt{ } T}(1-\theta)$

We used the fact that the density of a gas is proportional to the pressure, whereas the average gas molecule velocity is proportional to $\sqrt{ } T$. The rate of desorption, $\Phi_{d}$, is proportional to a frequency, $f$, at which the surface atoms vibrate (this frequency is of the order of $10^{13} \mathrm{~Hz}$ ) and the Boltzmann distribution factor, giving Equation (4), where $H_{a d}$ is the enthalpy of adsorption, $k$ is the Boltzmann constant, and $\theta$ is the fraction of occupied adsorption sites.

$\Phi \propto f e^{-\frac{H_{a d}}{k T} \theta}$

Combining these two gives Equation (5), in which $C$ is a constant.

$P=C f \sqrt{ } T e^{-\frac{H_{a d}}{k T}} \frac{\theta}{1-\theta}$

When the volume of the gas phase is small, we can assume the fractional coverage $\theta$ to be constant in the temperature range investigated. For two samples (SWNT and NAC) we recorded the pressure as a function of the sample temperature during cooling. The pressure of hydrogen gas above Norit GSX during warming was also measured. These measurements are presented in Figure 2. The least-squares fit on the GSX data with the model derived above is shown in the figure. Results from these fits are given in Table 1. From the errors associated with the fitted values of the adsorption potentials,

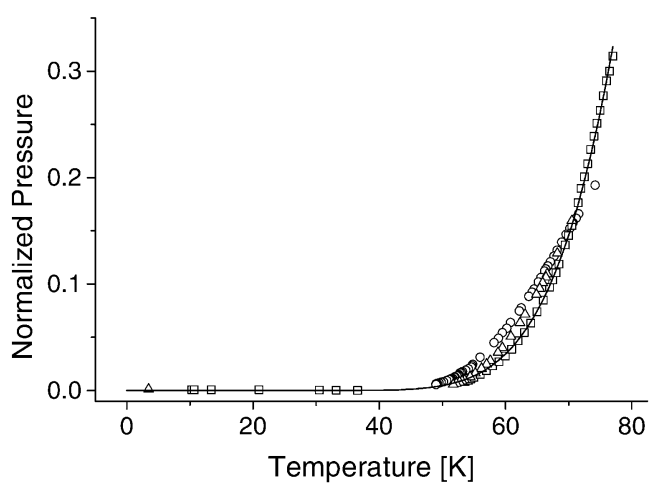

Figure 2. Temperature-pressure curves for SWNT (circles), Norit AC 990293 (triangles), and Norit GSX (squares). The line is a fit with the model

$P=C^{\prime} \sqrt{ } T e^{-\frac{H_{a d}}{k T}}$ through the measured curve for Norit GSX. Using this model the adsorption energies were obtained $(580 \pm 60 \mathrm{~K})$. 
Table 1. Properties of the materials investigated. $V$ is the volume at standard temperature and pressure (1 bar and $295 \mathrm{~K}$ ) of hydrogen stored in $1 \mathrm{~g}$ of material at $77 \mathrm{~K}$ and $1 \mathrm{bar}, S_{B E T}$ is the BET surface area (the value for SWNT is from ref. [31]) $H_{a d}$ is the enthalpy of adsorption derived from pressure-temperature curves and $p$ is the peak position in the neutron spectra due to the rotational transition.

\begin{tabular}{lrlcr}
\hline & NAC & GSX & SWNT & GNF \\
\hline$V[\mathrm{~mL} \mathrm{STP}]$ & 238 & 160 & 60 & 20 \\
$S_{B E T}\left[\mathrm{~m}^{2} \mathrm{~g}^{-1}\right]$ & 2200 & 933 & 380 & 196 \\
$H_{a d}[\mathrm{~K}]$ & 513 & 576 & 525 & - \\
$p\left[\mathrm{~cm}^{-1}\right]$ & 119 & 120 & 119 & 117 \\
\hline
\end{tabular}

we conclude that the adsorption potential is $580 \pm 60 \mathrm{~K}$ or $5 \pm$ $0.5 \mathrm{~kJ} \mathrm{~mol}^{-1}$. The review article of Vidali et al. on potentials of physical adsorption gives a well depth of $51.7 \mathrm{meV}$ for graphite, which equals $600 \mathrm{~K}$ or $5 \mathrm{~kJ} \mathrm{~mol}^{-1},{ }^{[25]}$ in agreement with our results. Clearly the value of Dillon et al. of $19.6 \mathrm{~kJ} \mathrm{~mol}^{-1}$ for hydrogen adsorbed onto single-walled carbon nanotubes is not reproduced. ${ }^{[1]}$

The macroscopic measurements show that hydrogen adsorption on carbon surfaces as a function of hydrogen pressure already starts to saturate at 1 bar at a temperature of $77 \mathrm{~K}$ (see Figure 1). The sites at which the hydrogen molecules adsorb under the experimental conditions in this work will be commensurate with the carbon hexagons in the matrix. ${ }^{[26]}$ The layout of these sites is hexagonal with a distance between reflections planes of $3.69 \AA \AA^{[26]}$ corresponding to a density of one hydrogen site per every three hexagons. This low density of sites explains how a coverage of $80 \%$ can be found from the Langmuir isotherm. On geometrical grounds, using a site area based on the hydrogen molecular size of $0.142 \mathrm{~nm}^{2}$ only, a coverage of about $34 \%$ is derived. ${ }^{[15]}$ It is known that at higher fillings (attainable using lower adsorption temperatures) an incommensurate hydrogen layer is formed with such a much higher packing. ${ }^{[26]}$

Inelastic neutron scattering results: Figure 3 shows the measured spectra for the four different carbon materials. Table 1 lists the results from least squares fits of the peaks with a Gaussian peak profile. The Gaussian peak profile was chosen because it is a good description of the resolution function of the instrument at these energy transfers. The determination of the absolute position of the peaks is influenced by systematic errors due to uncertainties in the neutron incident energy and path lengths in the instrument. We estimate this error to be $2 \%$, which is $2 \mathrm{~cm}^{-1}$, of the neutron energy loss. The fitted peak positions show that a shift of the rotational transition energy cannot be observed within experimental accuracy. The peak widths are comparable with the experimental resolution, which was determined to be $11 \mathrm{~cm}^{-1}$ (full width at half maximum) from a gauge measurement on solid hydrogen. No splitting of the $J=1$ level due to hindering of the rotation was observed.

The intensities in the elastic peak (zero-energy transfer) are much lower than the rotational transition peak. This excludes the presence of molecular ortho-hydrogen and of atomic hydrogen in significant amounts, because scattering cross-

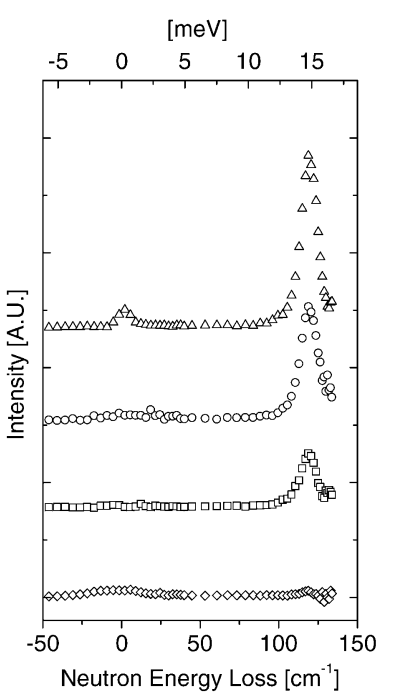

Figure 3. Neutron energy loss spectra of hydrogen adsorbed in Norit AC990293 (triangles), single-walled carbon nanotubes (circles), Norit GSX (squares), and graphitic nanofibers(diamonds). The data are normalized on the incident number of neutrons on the sample. The (mostly elastic) scattering of the sample without hydrogen has been subtracted (see text) which makes that only the scattering by hydrogen is visible. The graphitic nanofibers adsorb even after extra filling a very small amount of hydrogen, resulting in a very weak peak.

sections for both are larger than for the transition of $J=0$ to $J=1$. Furthermore, the elastic peak intensity compared to the inelastic peak intensity is as expected for the scattering by the para-hydrogen in the samples. Also when observing the spectra of the samples as a function of time (obtained from the different subruns for each spectrum) it was clear that the conversion of ortho- to para-hydrogen was already completed during the few hours of cooling. This is caused by the enhanced nuclear relaxation induced by the paramagnetic catalyst residuals (SWNT, GNF) or impurities (NAC, GSX).

Because the elastic peak intensity is so small this leaves no room for atomic hydrogen (having a large neutron crosssection) to be present in any significant amount. The assumption that a part of the reversible hydrogen storage is via the formation of atomic hydrogen and hydrogenation of the carbon is therefore not supported by the results. This contrasts with the assumptions in some recent theoretical works. ${ }^{[9]}$ Also in the Pd loaded sample there is no sign of enhanced $\mathrm{H}_{2}$ take-up due to the formation of atomic $\mathrm{H}$ (the $\mathrm{Pd}$ itself will absorb hydrogen up to $\mathrm{PdH}_{0.6}$, but the concentration is too low to observe).

The $J=1$ rotational peak is in the same position in carbon materials that have completely different topologies: the highly regular curved sheets of carbon in SWNTs, conical curved carbon sheets in fishbone nanofibers, and highly porous activated carbons. Evidently the conclusion has to be that the morphology of the carbon surfaces has little effect on the rotation potentials for hydrogen. This would also indicate that the bonds responsible for the adsorption are similar in the different materials, in agreement with the macroscopic adsorption data. The claims for large adsorption capacities in SWNTs can now be discarded both on the basis of 
macroscopic adsorption data and microscopic interaction strength's.

Discussion INS results: Here we will compare our neutron results on the SWNT with the three other types of sample as well as with results found by others in $\mathrm{C}_{60}{ }^{[22]}$ and single-walled nanotube containing samples. ${ }^{[27,28]}$ This comparison will give an answer to where the hydrogen molecules are located in the SWNT sample.

It is important to note that SWNT form bundles of many aligned tubes during the production process. ${ }^{[29]}$ On our sample we found that the X-ray diffraction pattern showed a peak at a momentum transfer of $0.536 \AA^{-1}$. This corresponds to a plane distance of $1.17 \mathrm{~nm}$, and assuming a close packing of the tubes, the average tube - tube distance is $1.35 \mathrm{~nm}$. This is close to the minimum distance between tubes that we measured using TEM $(1.25 \mathrm{~nm})$. We will use the X-ray value of $1.35 \mathrm{~nm}$ because it may represent a better average tube - tube distance for the bulk of the sample.

It is sometimes suggested that the hydrogen molecules go in the interstitial channels of the nanotube bundles. In that case the number of available hydrogen sites will be very large compared to that where hydrogen adsorbs on the outer surface of the bundles only. In $\mathrm{C}_{60}$ hydrogen indeed enters such interstitial sites. Fitzgerald et al. used a pressure of 130 bar to load hydrogen in $\mathrm{C}_{60}$ at room temperature, after which the hydrogen was frozen in the lattice at $150 \mathrm{~K}$. The position of the hydrogen molecules on the interstitial sites in $\mathrm{C}_{60}$ was determined by neutron diffraction methods. ${ }^{[22]}$ There are two types of sites (or voids) available for $\mathrm{H}_{2}$ : one with a diameter of $2.26 \AA$ and one with a diameter of $4.12 \AA$. Only the last site was shown to accommodate $\mathrm{H}_{2}$ up to $40 \%$, the first site is not occupied at all. The fact that the $\mathrm{H}_{2}$ does not enter the smaller site was explained by the size of the $\mathrm{H}_{2}$ molecule being too large, it has a diameter of $2.9 \AA$. The $J=1$ level of hydrogen in the occupied sites was shifted downwards to $14.35 \mathrm{meV}\left(115.7 \mathrm{~cm}^{-1}\right)$, and also split ${ }^{[22]}$ by $0.7 \mathrm{meV}\left(6 \mathrm{~cm}^{-1}\right)$. This indicates a significantly larger change in the rotational energy levels and also interaction of the hydrogen with the $\mathrm{C}_{60}$ matrix than in any of the samples measured by us. Such splittings and shifts would have been observed in our experiments. The fact that we find peaks that within error bars are not shifted with respect to solid hydrogen, shows that indeed hydrogen is very weakly disturbed by the bond to the surface of all of the carbon materials under investigation. This indicates a weak bond, which is supported by the macroscopic measurements.

Inelastic neutron spectra in samples containing soot and $20-30 \mathrm{wt} \%$ or $50 \mathrm{vol} \%$ of single-walled carbon nanotubes have been published before by Brown et al. ${ }^{[27]}$ and Ren et al., ${ }^{[28]}$ respectively. These samples contained significant amounts ( $80-70 \mathrm{wt} \%$ and $50 \mathrm{vol} \%$, respectively) of amorphous and nanocrystalline carbon as well as metal nanoparticles. Especially the nanostructured carbon is problematic in this respect because its surface area will be non-negligible, making significant amounts of hydrogen adsorption possible. Their experiments used pressures as high as 110 bar to load the sample container with the sample with hydrogen. After this initial loading the sample was cooled to $25 \mathrm{~K}$, where the hydrogen source was disconnected. Subsequently the cells were evacuated at about $25 \mathrm{~K}$ to remove any non-adsorbed hydrogen. It may be noted that this loading procedure is rather different from ours, because we used just 1 bar at $77 \mathrm{~K}$ to adsorb the hydrogen. However, we obtained in a reproducible manner similar or higher adsorbed hydrogen amounts for the SWNT. Both articles report the inelastic (unsplit) peak due to the hydrogen molecular rotor. The peak position was found to be close to the value for the free $\mathrm{H}_{2}$, the elastic peak was not clearly shown.

Combining the results and comparing with the situation for $\mathrm{C}_{60}$, we may state that $\mathrm{H}_{2}$ does not enter the interstitial channels of the SWNT sample, and the reason why may be illustrated in Figure 4. The diameter of the interstitial

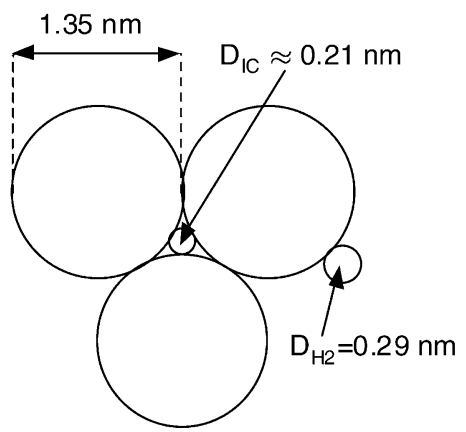

Figure 4. Schematic graph of the consequence of the stacking of nanotubes in bundles with tube-tube distances of $1.35 \mathrm{~nm}$. The interstitial channel between the bundles is indicated to be too small for hydrogen molecules (2.1 $\AA$ versus $2.9 \AA)$.

channels between nanotubes of $1.35 \mathrm{~nm}$ diameter is $2.1 \AA$, which is too small for hydrogen molecules to enter. It is even smaller than the void in $\mathrm{C}_{60}$ of $2.26 \AA$ which is not occupied by $\mathrm{H}_{2}$ either. Furthermore the inelastic neutron data show no difference between the four different types of nanostructured carbon samples. Because the size of the channel between the SWNTs is much smaller $(2.1 \AA)$ than the void in $\mathrm{C}_{60}(4.12 \AA)$ one would expect at least a similar but likely a larger influence on the spectra of the adsorption in the channels. Clearly this is not the case and we therefore have to exclude that significant amounts of $\mathrm{H}_{2}$ adsorbs in the interstitial channels. It may also be noted that Talapatra et al. reported that smaller gases like $\mathrm{He}$ and $\mathrm{Ne}$ did not enter the interstitial channels either. ${ }^{[29]}$

Calculations: The materials all have aromatic rings in their graphite structure. The fact that the different samples give the same results for the interaction potentials raises the hypothesis that the characteristic van der Waals bonding can be modelled by only taking into account a carbon hexagon structure with its delocalized electrons.

As a check we performed DFT calculations using Dmol ${ }^{3}$. We used the numerical basis set DND (double numerical with $\mathrm{d}$ functions) and the Perdew Wang local correlation density functional. First we optimized the position of a hydrogen molecule above a small piece of graphite built from four carbon hexagons (Figure 5). The position with lowest energy for the hydrogen molecule was $2.91 \AA$ above a central $\mathrm{C}-\mathrm{C}$ bond. Then we turned the hydrogen molecule stepwise by 


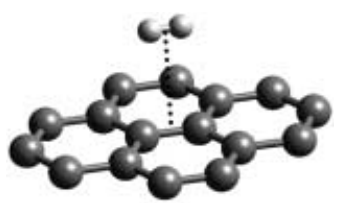

Figure 5. The graphite surface and the hydrogen molecule in their minimum energy position. The hydrogen molecule is located right above the center of the $\mathrm{C}-\mathrm{C}$ bond. From this configuration the energy was calculated by using DFT when the hydrogen molecule was turned around.

$180^{\circ}$ and evaluated the potential energy each $20^{\circ}$. In this way we obtained an estimate of the barrier for rotation for the adsorbed molecule. The barrier we find is indeed very small and is within computational accuracy consistent with the experiments.

We conclude that different forms of carbon are essentially the same for hydrogen molecules as long as they possess the characteristic aromatic carbon rings. Thus the amount of hydrogen storage is governed by the number of accessible aromatic $\mathrm{C}-\mathrm{C}$ bonds in the sample, which is related to the surface area. The morphology of the adsorption sites on more than one $\mathrm{C}-\mathrm{C}$ bond length is less important.

\section{Discussion}

From this study some perspectives for hydrogen storage using physisorption onto graphite surfaces can be evaluated. It appears that these materials will not meet the goals set by the Department of Energy of the US (6.5 wt \%) under moderate conditions such as room temperature and pressures up to 10 bar. This can be concluded from the interaction energy which is around $580 \mathrm{~K}\left(5 \mathrm{~kJ} \mathrm{~mol}^{-1}\right)$ (which means that at room temperature virtually no hydrogen adsorbs) and from the fact that even a single graphite sheet needs to be covered for $80 \%$ by hydrogen molecules on both sides to meet the goals.

An ideal hydrogen storage material using physisorption would have an interaction potential around $2500 \mathrm{~K}$ $\left(\approx 20 \mathrm{~kJ} \mathrm{~mol}^{-1}\right)$ and a very high surface area $\left(>2000 \mathrm{~m}^{2} \mathrm{~g}^{-1}\right)$. In this way the residence time at room temperature for adsorbed molecules is comparable with hydrogen adsorbed on graphite at $77 \mathrm{~K}$. Optimizing the surface area and hydrogen pressure could then in principle lead to a material capable of storing $6.5 \mathrm{wt} \%$ of hydrogen at room temperature and moderate pressures. However, at present there is no indication as how to realize such a high interaction potential in a material with a high specific surface area.

Recent experiments on carbon materials by G. Gundiah and co-workers showed hydrogen storage capacities in the range of $0.2 \mathrm{wt} \%$ to $3.7 \mathrm{wt} \%$ at $300 \mathrm{~K}$ and 140 bar. $^{[30]}$ To compare these results with ours, we have to extrapolate the use of Equation (5) to a temperature of $300 \mathrm{~K}$. We take the coverage $\theta$ to be the same for both temperatures, meaning the same amount of storage. Further we assume the frequency $f$ and the adsorption enthalpy $H_{a d}=580 \mathrm{~K}$ to be constant. With these assumptions we estimate that a pressure of 530 bar at $300 \mathrm{~K}$ will give similar storage capacities as 1 bar at $77 \mathrm{~K}$. In our work we found a hydrogen storage capacity for SWNT of $60 \mathrm{~mL}$ STP per $\mathrm{g}$, which equals $0.54 \mathrm{wt} \%$. The ratio of the estimated pressure (530 bar) and the pressure used by Gundiah et al. (140 bar) is comparable to the ratio in hydrogen storage capacities $(0.54 \mathrm{wt} \%$ versus $0.2 \mathrm{wt} \%$ for untreated SWNT). Their results for untreated SWNT therefore support the findings presented in this work.

\section{Conclusions}

We report a study of microscopic aspects of physisorption of hydrogen on carbon surfaces with several nano-morphologies. From the isotherms and temperature dependent pressure measurements we conclude that the interaction between a hydrogen molecule and a carbon surface is $580 \pm 60 \mathrm{~K}$ or $5 \pm$ $0.5 \mathrm{~kJ} \mathrm{~mol}^{-1}$. However, at room temperature and ambient pressure the residence time is very short, leading to negligible adsorption. Inelastic neutron scattering experiments reveal that molecular $\mathrm{H}_{2}$ is present and that the splitting of the ground and excited level of the molecular hydrogen rotor is essentially the same as for free hydrogen in all investigated carbon materials. No sign of a strong bond of the hydrogen molecule with the substrate was found in any of the materials. This finding compared to $\mathrm{H}_{2}$ loaded interstitially in $\mathrm{C}_{60}$, and the low adsorption quantities, rule out the possibility that $\mathrm{H}_{2}$ adsorbs in the narrow interstitial channels between the nanotubes. The bond of hydrogen to carbon does not depend on the topology of the surface. The only thing that counts then is the amount of surface accessible by hydrogen. This surface area appears to be largest in the activated carbons. The SWNTs form bundles and this aggregation apparently severely limits the accessible surface. Results of DFT calculations confirm that the hydrogen molecule is weakly bound to a $\mathrm{C}-\mathrm{C}$ bond where it experiences only a small barrier for rotation.

\section{Acknowledgement}

This work is part of the Delft Institute for Sustainable Energy (DISE). We thank Mr. C.F. de Vroege for assistance during neutron scattering experiments, M. W. J. Crajé for the Mössbauer experiments and Mr. F. D. Tichelaar for the TEM images.

[1] A. C. Dillon, K. M. Jones, T. A. Bekkedahl, C. H. Klang, D. S. Bethune, M. J. Heben. Nature, 1997, 386, 377.

[2] A. Chambers, C. Park, R. T. K. Baker, N. M. Rodriguez, J. Phys. Chem. B, 1998, 102, 4253.

[3] C. Park, P. E. Anderson, A. Chambers, C. D. Tan, R. Hidalgo, N. M. Rodriguez. J. Phys. Chem. B, 1999, 103, 10572.

[4] P. Chen, X. Wu, J. Lin, K. L. Tan. Science, 1999, 285, 91.

[5] Y. Ye C. C. Ahn, B. V. Ratnakumar, C. Witham, Jr. R. C. Bowman, B. Fultz. Appl. Phys. Lett. 1998, 73, 3378.

[6] F. E. Pinkerton, B. G. Wicke, C. H. Olk, G. G. Tibbets, G. P. Meisner, M. S. Meyer, J. F. Herbst. J. Phys. Chem. B, 2000, 104, 9460.

[7] M. Hirscher, M. Becher, M. Haluska, U. Dettlaff-Weglikowska, A. Quintel, G. S. Duesberg, Y.-M. Choi, P. Downes, M. Hulman, S. Roth, I. Stepanek, P. Bernier. Appl. Phys. A, 2001, 72, 129.

[8] M. Hirscher, M. Becher, M. Haluska, A. Quintel, V. Skakalova, Y.-M. Choi, U. Dettlaff-Weglikowska, S. Roth, I. Stepanek, P. Bernier, A. Leonhardt, J. Fink, J. All. Comp. 2002, 330-332, 654.

[9] V. Meregalli, M. Parrinello. Appl. Phys. A, 2001, 72, 143. 
[10] H. Cheng, G. Pez, G. Kern, G. Kresse, J. Hafner, J. Phys. Chem. B, 2001, 105, 736.

[11] R. F. Cracknell, Phys. Chem. Chem. Phys., 2001, 3, 2091.

[12] P. A. Gordon, R. B. Saeger. Ind. Eng. Chem. Res., 1999, 38, 4647.

[13] Q. Wang, S. R. Challa, D. S. Sholl, J. K. Johnson. Phys. Rev. Lett. 1999, $82,956$.

[14] a) H. Dodziuk, G. Dolgonos, Chem. Phys. Lett., 2002, 356, 79; b) M. Rzepka, P. Lamp, M. A. de la Casa-Lillo, J. Phys. Chem. B, 1998, 102, 10894.

[15] M. G. Nijkamp, J. E. M. J. Raaymakers, A. J. van Dillen, K. P. de Jong. Appl. Phys. A, 2001, 72, 619.

[16] The energy of adsorption is defined to be smaller than $4000 \mathrm{~K}$ for physisorption. ${ }^{[17]}$ If the energy of adsorption is greater than $4000 \mathrm{~K}$ the molecule forms chemical bonds.

[17] G. Attard, C. Barnes, Surfaces, Oxford University Press, Oxford, 1998.

[18] M. L. Toebes, J. H. Bitter, A. J. van Dillen, K. P. de Jong, Cat. Today, 2002, 76, 33.

[19] B. L. Mojet, M. S. Hoogenraad, A. J. van Dillen, J. W. Geus, D. C. Koningsberger, J. Chem. Soc. Faraday. Trans. 1997, 93, 4371.

[20] W.-F. Du, L. Wilson, J. Ripmeester, R. Dutrisac, B. Simard, S. Dénommée, Nano Lett. 2002, 2, 343.
[21] I. F. Silvera. Rev. Mod. Phys. 1980, 52, 393.

[22] S. A. Fitzgerald, T. Yildirim, L. J. Saltodonato, D. A. Neumann, J. R. D. Copley, J. J. Rush, F. Trouw. Phys. Rev. B, 1999, 60, 6439.

[23] J. Eckert, G. J. Kubas, J. Phys. Chem., 1993, 97, 2378.

[24] B. L. Mojet, J. Eckert, R. A. van Santen, A. Albinati, R. E. Lechner, J. Am. Chem. Soc. 2001, 123, 8147.

[25] G. Vidali, G. Ihm, H.-Y. Kim, M. W. Cole, Surf. Sci. Rep. 1991, 12, 135.

[26] M. Nielsen, W. Ellenson, Proc. 14th Int. Conf. Low Temp. Phys. 1975, $4,437$.

[27] C. M. Brown, T. Yildirim, D. A. Neumann, M. J. Heben, T. Genett, A. C. Dillon, J. L. Alleman, J. E. Fischer, Chem. Phys. Lett., 2000, 329, 311.

[28] Y. Ren, D. L. Price. Appl. Phys. Lett., 2001, 79, 3684.

[29] S. Talapatra, A. Z. Zambano, S. E. Weber, and A. D. Migone. Phys. Rev. Lett. 2000, 85, 138

[30] G. Gundiah, A. Govindaraj, N. Rajalakshmi, K. S. Dhathathreyan, C. N. R. Rao, J. Mat. Chem. 2003, 13, 209.

[31] M. Eswaramoorthy, R. Sen, C. N. R. Rao, Chem. Phys. Lett. 1999, 304, 207.

Received: February 12, 2003 [F 4845] 